BENTHAM OPEN

\title{
A Multi-Type Queuing Network Analysis Method for Controlling Server Number in the Outpatient
}

\author{
Zhu Mingzhu ${ }^{*}$ and Qi Ershi \\ College of Management and Economics, Tianjin University, Tianjin, 300072, P.R. China
}

Received: August 16, 2015

Revised: September 16, 2015

Accepted: October 1, 2015

\begin{abstract}
Patients long time waiting is the most significant problem in Chinese outpatient, and the inadequate service level, as one of main factors, causes the medical error and outpatient overcrowding. This paper derives a multi-type queuing network model to help managers increase service efficiency and improve patient satisfaction based on Chinese current outpatient condition. One of this model's characteristics is capturing different patient types who pass through the system in different service routes. The other unique thing is taking the outpatient service process as a whole queuing network with multi-type patients, not a simple tandem queuing network. Only by the external arrival rate, the arrival rate of each service node can be calculated in order to obtain the system operation indicators including server utilization, waiting time and patient number in queue and system as the service targets, in this way, the hospital manager can clarify the current outpatient operation and promote the situation by modifying the server number. Results from one actual case are used to demonstrate the methodology. This multi-type queuing network analysis method can assist to organize the server resource more effectively in the status of limited resource and excessive demand, and can be an important innovation in hospital management.
\end{abstract}

Keywords: Mathematical models, multi-type patients, multi-type queuing network, outpatient process, server number.

\section{INTRODUCTION}

There is a serious queuing phenomenon in Chinese health care due to the inadequate resource of health care and the high demand for health care, typically in the outpatient. Patient arrivals in the outpatient cannot be scheduled or controlled significantly because fewer patients choose to make an appointment with doctors in China. The unique characteristic in Chinese outpatient is "three-long and one-short" (long-time queuing for the registration and paying, long-time waiting for doctors' consultation, long-time waiting for the examination and short-time doctors' consultation), commonly in the outpatient of Chinese large general hospitals [1]. During the whole process of medical service, the average waiting time in the outpatient is 1-1.5 hours in general, whereas the doctor's diagnosis time is only 10-15 minutes. Patients spend a lot of time on non-medical activities [2]. This phenomenon leads to three social issues: (1) The patient satisfaction decreases obviously because patients with physical discomfort spend hours in crowded waiting room to see a doctor, and patients take the risk of worsening their condition and security in the crowd [3]; (2) The staff endures the overloading work stress, which decreases their work efficiency, quality and makes them more susceptible to medical errors [4]; (3) The deterioration in relations between patients and staff results in more dispute easily, even the adverse event of hurting medical staff by patients. The medical problems occurring in China have been intensified with more medical disputes between medical staff and patients [5].

Health care managers face a challenging task to organize the resource more effectively and efficiently in the state of limited medical resources and the excess demand. Patients certainly hope to wait in the outpatient as short a time as possible and to improve their satisfaction by increasing the capacity of the service facility. On the other hand, the managers desire to use the resource as lest as possible and to meet the patients need effectively. The key of this problem

\footnotetext{
* Address correspondence to this author at the Tianjan University, No. 92, Weijin Road, Nankai District, Tianjin, 300072, P.R. China; Tel: +86-158 220793 84; E-mail: dorothyhatty@126.com
} 
compromises between excessive need and limited resource. The common methods to solve this problem, including Business Process Reengineering, Six Sigma and Lean Thinking, are used in the process design or the case analysis, so this kind of method only analyzes the specific process in special context. In addition, the simulation model as a usual method also can describe the complex dynamic behavior as accurately as the queuing model, but the drawbacks of simulation are the extensive data and the prohibitive expense associated with such data collection [6, 7]. Therefore, the queuing theory has advantages in dynamic behavior description and data collection compared with other methods. The approach of queuing theory, based on mathematical models, can successfully address the problems in health care system and assist the hospital manager to make decisions. The queuing theory can simulate the actual structure and behavior scientifically, access the optimal evaluation, meet the needs of patients to the greatest extent, and find a balance point between service quality and service cost so as to achieve the maximum economic interests and social benefits $[8,9]$.

Most of the queuing theory researches in health care modeled the health care system as a single server node and studied each service node within the outpatient process in separation. Queuing network models are less common and the majority of queuing network models in health care is for limited networks such as a tandem queuing network that the patients are entered each service node in the same order, not considering the different type of patients who visit service nodes in different orders. The purpose of this paper is to establish a multi-type queuing network model. Each type represents a different patient type who visits service nodes in one order. The model contains all the service nodes in the whole outpatient process and then to allocate the number of servers in the outpatient resource, based on the queuing network theory.

The remainder of the paper is organized as follows. Section 2 presents a literature review of the research on the outpatient with the queuing theory and points out the difference between Chinese and foreign researches. Combined with the actual situation of Chinese outpatient, Section 3 sets up a multi-type queuing network model and introduces how four different kinds of patients are routed in the model. Section 4 defines the mathematical methodology of the model to evaluate the operation indicators of each node in the outpatient. Section 5 presents a case study in the use of the mathematical methodology to complete the optimizing allocation of server number. Section 6 concludes that the queuing network model conforms to the actual demand for Chinese hospital service and proposes the limitations for future improvement.

\section{LITERATURE REVIEW}

The crowded phenomenon in the health care of most countries in the world happens more seriously and prevalently, deserving more immediate attention. In recent years, the queuing theory is introduced into the field of medical service and combined with the information system, which become the practical solution to tackle the queuing problem and relieve the crowd in health care. The queuing theory has its advantage in producing the simple models especially for the application of less random data. It is easy to construct a queuing model for a large general hospital [10]. The queuing theory also can be applied for the rapid evaluation and the comparison among various alternatives. Consequently, the queuing model becomes a powerful tool for the outpatient research. Queuing theory can solve the basic problem of maximum capacity to associate with a specific service standard in the health care, allocate rationally and effectively various service resources like facility, staff, space etc., and choose the priority queuing discipline to determine service order among patients [11]. Therefore, queuing theory is widely used in the academic research and practical application on the service system.

The service process of foreign outpatients has a significant difference with Chinese outpatient process. In the foreign outpatients, there is almost no queuing phenomenon because most foreign outpatients provide the reservation system for the patients. For this reason, the queuing theory in foreign researches is mostly applied in the emergency, inpatient, operating room and other departments. Mehandiratta [12] studied the application of queuing theory in the medical system and presented its efficiency in the inpatient, office, public health, emergency preparedness, long-term care and so on. Fomundam and Herrmann [13] analyzed the medical resource allocation based on the queuing theory and summarized the contribution of queuing theory in the health care. Cochran and Roche [14] derived an open queuing network model of an emergency department design intended to increase the capacity of emergency department to treat patients according to the patient acuity, arrival patterns and volumes. Yankovic and Green [15] represented the nursing system as a variable finite-source queuing model and developed a reliable, tractable, easily parameterized twodimensional model to approximate the actual interdependent dynamics of bed occupancy levels and demands for nursing. 
Among Chinese researches on outpatient service problems with the queuing theory, a part of the researches looked upon the outpatient process as a tandem queuing network that the patients entered each service node orderly within the outpatient, not considering the state that some patients visit some nodes repeatedly. Mi [16] and Feng [17] both researched the outpatient queuing network according to the registration, diagnosis, paying and taking medicine in sequence, only once visit on one node. Another part of the Chinese literatures studied each service node within the outpatient process in separation and analyzed the queuing indicators of each node respectively. Chang et al. [18] and Zhou [19] studied the service efficiency of outpatient registration and cashier separately with the queuing theory. In contrast, this paper takes the outpatient service process as a whole queuing network and calculates the performance metrics of each node only by the external arrival rate of patients. This paper also considers the different types of patients accepting the different service orders and focuses on the reciprocating movement of patients among the service nodes in line with the actual outpatient circumstance.

\section{OUTPATIENT PROCESS DESCRIPTION}

The general service process in Chinese outpatient consists of five service nodes: registration, consulting room, cashier, laboratory and pharmacy (including the injected patients those go to the pharmacy to take the injection). These five nodes are assigned from 1 to 5 , and 0 means to leaving from the outpatient.

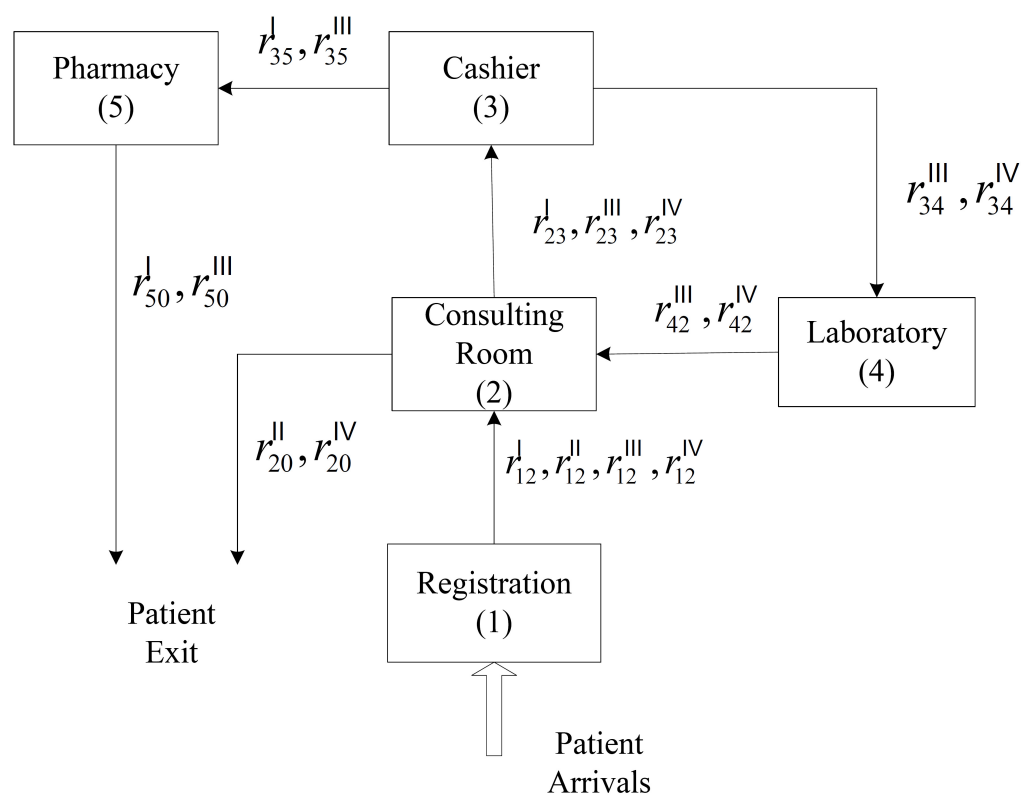

Fig. (1). Outpatient process network $\left(r_{i j}^{t}\right.$ represents the probability of patient-flow from node $i$ to node $j$ for patient type $t$ ).

As shown in Fig. (1), all the patients arrive at the registration firstly, and then go to the consulting room to see the doctor. After the doctor knows about the patient condition preliminarily, one parts of patients can be diagnosed directly, those the doctor regards to get the mild disease. They can continue to go to the cashier to pay with the doctor's prescription, get the medicine in the pharmacy and leave the outpatient finally. This kind of patients is set for Type I. There are also a few special cases in this part of patients. Sometimes, they do not need any treatment after the doctor's diagnosis and leave directly, or they are not willing to accept the doctor's prescription and leave. Type II is for such patients.

Another part of patients' condition is more complex, and the doctor needs more accurate examination to diagnose for this kind of patients. So the doctor asks patients to do some inspection. The patient goes to the cashier to pay the inspection item firstly, and then the patient continues to reach the laboratory to check up like blood test, X-ray, B ultrasonic, electrocardiogram, radiography, etc. After obtaining the inspection report, the patient returns to the consulting room. The doctor makes the relatively accurate diagnosis for the patient combing with his examination result. Typically, this kind of patients goes to the cashier again after the doctor's diagnosis, takes the medicine in the pharmacy and leaves the outpatient finally. This kind of patients is set for Type III. Although a few patients also accept 
the initial diagnosis from the doctor, they change their mind to give up the further treatment or not agree with this doctor's diagnosis for some reason and leave the outpatient at last. Type IV is for such patients. Here, the patients need to be hospitalized are regarded as leaving from the outpatient service system.

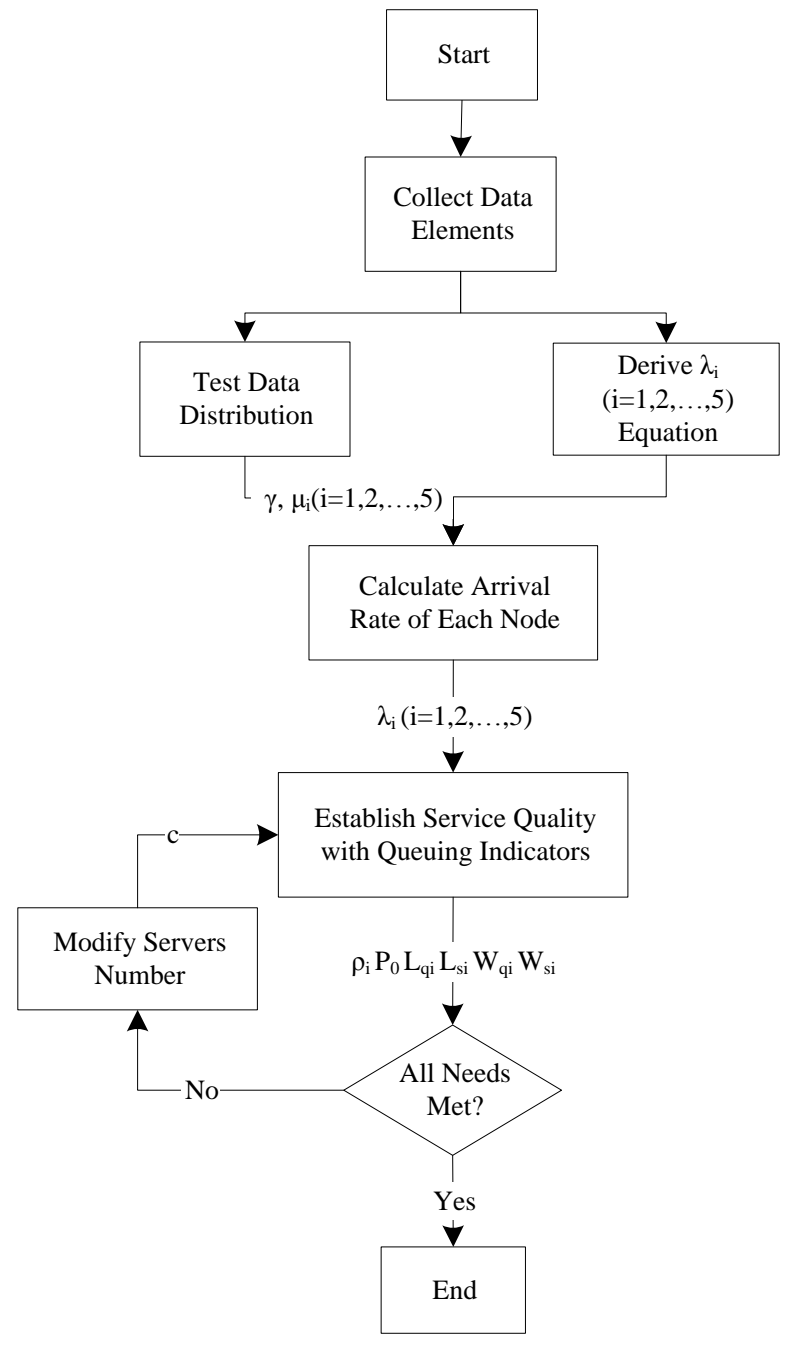

Fig. (2). The methodology for optimizing outpatient service.

\section{MATHEMATICAL METHODOLOGY}

Queuing network is composed of several random queue systems, mostly limited and single queue systems. Different types of customers pass through the network in different ways or routes and are served by the service nodes within the network. A queuing network includes a set of nodes $(i)$. Each node has a number of servers $(c)$ and a single node can be regarded as a queuing system. Customers can access to the queuing network from any node. The arrival rate from the outside is $\gamma$ and the arrival rate of Node $i$ is $\lambda_{i}$. After the customer queues and gets the service at a certain node (the service rate of Node $i$ is $\mu_{i}$ ), he can leave this network or go to another node, or even return to the original node. In the outpatient, the patient's arrival, wait, consultation and leaving can be viewed as a queuing network system. Fig. (2) displays the methodology to analyze the outpatient process, to establish service quality by the performance indicators (server utilization $\rho$, the idle probability of staff $P_{0}$, queue length $L_{q}$, customer number in the system $L_{s}$, waiting time in queue $W_{q}$ and system $W_{s}$ ) and to modify the server number $(c)$ to meet an acceptable service level.

\subsection{Primary Data Analysis}

After collecting primary data elements, it is necessary to make the following assumptions of arrival time, service time, server arrangement, customer resource, and service rule for the queuing network model. 
1. Arrival time distribution: patients those arrive at the outpatient from the outside usually go to the registration first, then to see the doctor. In this process, the arrival time of each patient among disjoint time intervals is independent of each other, and only one patient can reach at any instant, being of the stability. In consequent, most previous researches regarded that the arrival time of patients conformed to Poisson distribution [20]. The distribution of primary data also can be proved by goodness-of-fit testing $\left(\chi^{2}\right.$ hypothesis test).

According to one of characteristics of the queuing network, the actual input of one node is the sum of arriving customers from the external network and other nodes within the network. A group of customers from one node will be split into several parts, and then, they enter into other nodes respectively or leave the network system directly. This merger or split of customers usually changes the input distribution of each node. However, if the external arrival process of all nodes is Poisson distribution, the type of distribution will remain during the merge or split, only the parameter change of Poisson distribution [21]. Therefore, if the external arrival process can be validated to be the Poisson distribution, the arrival process to other node can be also regarded as the Poisson distribution.

2. Service time distribution: patients pass through each node in turn, all service time is independent of each other following the Exponential distribution, which also can be proved by $\chi^{2}$ test.

3. Server arrangement: several servers for patients will be arranged on every node at usual, so the queuing system on each node is treated as a system of multiple servers in parallel.

4. Capacity: the outpatient never stops patients to be served in general. The situation, that patients are restricted to entering into the outpatient due to excess number, rarely occurs. The service capacity of the queuing system is taken as infinite.

5. Amount of resource: all patients in the outpatient are customer source of this queuing system, so patient resource in the outpatient can be viewed as infinite.

6. Queuing discipline: queuing discipline is usually first come first served in the outpatient.

\subsection{Calculate Arrival Rate of Each Node}

How to calculate the arrival rate of all nodes should be considered after the primary data analysis. In a queuing network with $k$ nodes, there are $c_{i}$ servers on each node $i(i=1,2, \ldots, k)$. The service time on individual customer is exponentially distributed, and the average service rate is $\mu_{i}$. Customers arrive at Node $i$ from the outside at the rate $\gamma_{i}$. The arrival process from the outside is Poisson distribution and the arrival rate of each node is $\lambda_{i}$. When a customer finishes his service in Node $i$, he enters another node $j(j=1,2, \ldots, k)$ and leaves the network system at the probability $r_{i j}$. All the service nodes can contain an unlimited number of customers. The probability of the customer transferring from one node to another has been unrelated to the service process or the servers in other nodes when this customer has been served. Such a queuing network is called Jackson queuing network. If the customers from the outside system are allowed to enter the network system and to leave the system at any moment, this network system is an open Jackson queuing network system. The rule of network path $\left\{r_{i j}, i(i=1,2, \ldots, k) ; j(j=1,2, \ldots, k)\right\}$ in Jackson queuing network conforms to $\sum_{i \quad 0}^{k} r_{i j}=1 i, j=1,2, \ldots, k$.

The calculation method of an open Jackson queuing system is to determine the arrival rate of customers in each node firstly, and then to process each node in accordance with an independent $\mathrm{M} / \mathrm{M} / \mathrm{c}$ queuing system $(\mathrm{M} / \mathrm{M} / \mathrm{c}$ represents a queuing system with the arrival process of Poisson distribution, the service time of Exponential distribution and $\mathrm{c}$ servers). The arrival rate of customers on every node should be equal to the leaving rate in a stable system. The arrival rate of one node should be equal to the sum of the external arrival rate from the outside system and internal arrival rate from other nodes [22]. Therefore, the arrival rate of each node $\lambda_{i}$ is calculated by Equation (1).

$$
\lambda_{i}=\gamma_{i}+\sum_{j=1}^{k} r_{j i} \lambda_{j} \quad i, j=1,2, \ldots, k \quad \ldots
$$

Most of previous queuing network researchers in health care only consider that the patients are entered each service node in the same order, so they take a tandem outpatient queuing network as an open Jackson queuing network and apply Equation (1) to solve the arrival rate of each node. The outpatient system researched in this paper does not conform to a Jackson queuing network because both Type II and Type IV patients return to the previous visited nodes, through the consulting room twice as shown in Fig. (2). This condition does not conform one of Jackson queuing network conditions: the probability of the customer transferring from one node to another has been unrelated to the 
service process and the server in other nodes when this customer has been served. To solve this kind of queuing network, firstly, it needs to be simulated or approximated a Jackson queuing network. It is assumed that the arrival rate is doubled; the random routes in the network are approximated several fixed routes to obtain the transition probability matrix of the network system. To calculate the arrival rate of each node, the product is firstly calculated between the times one route through this node and the external input rate of this route, secondly, sum the product of each route [23]. For instance, one queuing network system with $k$ nodes is approximated $n$ fixed routes. If $\gamma_{m i}$ is the external arrival rate of Route $m$ on Node $i$ and $\eta_{m i}$ is the times Route $m$ through Node $i$, the total arrival rate of Node $i$ is calculated by Equation (2).

$$
\lambda_{i}=\sum_{m=1}^{n} \gamma_{m} \eta_{m i} \quad i=1,2, \ldots, k ; m=1,2, \ldots, n
$$

Using Equation (2), the total arrival rate to one node in this kind of queuing network can be obtained.

As shown in Fig. (1), there are four fixed routes, namely four types of patients:

Type I: 1-2-3-5

Type II: $1-2$

Type III: 1-2-3-4-2-3-5

Type IV: 1-2-3-4-2

Here, 1 to 5 represents five service nodes separately. Table 1 shows the times each route through each node and the equation of total arrival rate to each node.

Table 1. Arrival rate of each node.

\begin{tabular}{|c|c|c|c|c|c|}
\hline & I & II & III & IV & $\lambda_{\boldsymbol{i}}$ \\
\hline Registration(1) & 1 & 1 & 1 & 1 & $\lambda_{1}=\gamma_{1}+\gamma_{2}+\gamma_{3}+\gamma_{4}$ \\
\hline Consulting Room (2) & 1 & 1 & 2 & 2 & $\lambda_{2}=\gamma_{1}+\gamma_{2}+2 \gamma_{3}+2 \gamma_{4}$ \\
\hline Cashier(3) & 1 & 0 & 2 & 1 & $\lambda_{3}=\gamma_{1}+2 \gamma_{3}+\gamma_{4}$ \\
\hline Laboratory(4) & 0 & 0 & 1 & 1 & $\lambda_{4}=\gamma_{3}+\gamma_{4}$ \\
\hline Pharmacy(5) & 1 & 0 & 1 & 0 & $\lambda_{5}=\gamma_{1}+\gamma_{3}$ \\
\hline
\end{tabular}

\subsection{Establish Service Quality}

As is known from the primary data analysis in Section 4.1, the small service system on each node in the outpatient is still equivalent to an $\mathrm{M} / \mathrm{M} / \mathrm{c}$ queuing system, because of the independent arrival process following Poisson distribution, the independent service time following Exponential distribution, $c_{i}$ servers on Node $i$, infinite patient resource, unlimited capacity in the system and first come first served queuing discipline.

Table 2 demonstrates the main parameters to measure the system performance and their calculated formulas as an $\mathrm{M} / \mathrm{M} / \mathrm{c}$ queue system. The patients take a concern of patient number in queue $\left(L_{q}\right)$ and in the system $\left(L_{s}\right)$, waiting time in queue $\left(W_{q}\right)$ and in the system $\left(W_{s}\right)$. The staffs mainly care about their idle proportion $\left(P_{0}\right)$. The managers of hospitals focus on not only the issues both patients and staffs concern but also server utilization. They need to consider the queuing system design and control after the performance analysis of current queuing system. These performance metrics can reflect the current status of the queuing system directly and provide a clear reference for the managers to adjust and control the system further. A high effectiveness of service will be provided for patients as the manager desire. The other performance metrics may be considered in future research.

Table 2. Performance metrics.

\begin{tabular}{|c|c|}
\hline Metrics & Formula \\
\hline Server utilization $(\rho)$ & $\rho=\frac{\lambda}{c \mu}$ \\
\hline Idle probability of staff $\left(P_{0}\right)$ & $\left.P_{0}=\left[\sum_{k=0}^{c-1} \frac{1}{k !}\left(\frac{\lambda}{\mu}\right)^{k}+\frac{1}{c !} \frac{1}{1-\rho} \square_{\mu}^{\lambda}\right]^{c}\right]^{-1}$ \\
\hline
\end{tabular}


(Table 2) contd.....

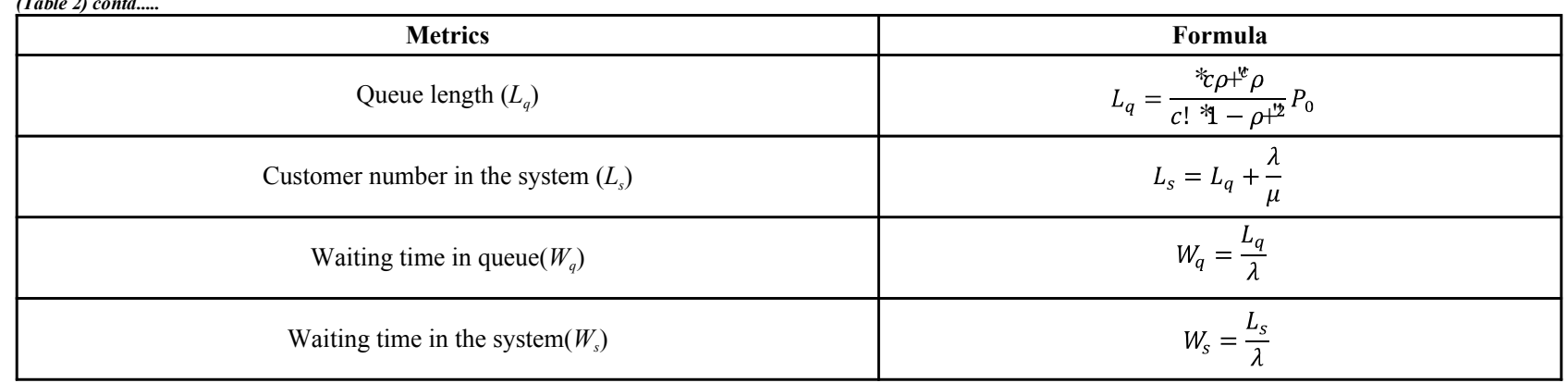

\section{CASE STUDY}

The primary data is collected from one large general hospital in Tianjin. The main data elements are observed and recorded in the field including the patient arrival time at the registration and the service time of five service nodes.

Table 3. Arrival time calculation of $\chi^{2}$ test statistics.

\begin{tabular}{|c|c|c|c|c|c|c|}
\hline$i$ & $x$ & $\mathbf{f}_{\mathrm{i}}$ & $\mathbf{p}_{\mathrm{i}}$ & $n p_{i}$ & $\left(\mathbf{f}_{\mathrm{i}}-\mathbf{n} \mathbf{p}_{\mathrm{i}}\right)$ & $\frac{\left(f_{i}-n p_{i}\right)^{2}}{n p_{i}}$ \\
\hline 1 & 0 & 38 & 0.069 & 29.183 & 8.817 & 2.664 \\
\hline 2 & 1 & 86 & 0.185 & 77.821 & 8.179 & 0.860 \\
\hline 3 & 2 & 90 & 0.247 & 103.762 & -13.762 & 1.825 \\
\hline 4 & 3 & 82 & 0.220 & 92.233 & -10.233 & 1.135 \\
\hline 5 & 4 & 55 & 0.146 & 61.489 & -6.489 & 0.685 \\
\hline 6 & 5 & 41 & 0.078 & 32.794 & 8.206 & 2.053 \\
\hline 7 & 6 & 16 & 0.035 & 14.575 & 1.425 & 0.139 \\
\hline 8 & 7 & 9 & 0.013 & 5.552 & 3.448 & 2.141 \\
\hline 9 & 8 above & 3 & 0.006 & 2.591 & 0.409 & 0.065 \\
\hline & Total & 420 & 1.000 & 420.000 & 0.000 & 11.567 \\
\hline
\end{tabular}

$i$ is each interval; $x$ is the arrival number per minute

\subsection{Primary Data Analysis}

The arrival process, that patients arrive at the registration in working hours ( 8 hours) of one day, is observed randomly. There are almost no patients arriving in the outpatient over the last working hour of the afternoon, hence, so the real observed time is totally 7 hours (420 minutes) and the total number of arrival patients is 1120 during this time. The arrival number per minute is recorded shown in Table $\mathbf{3}$ to calculate the minutely arrivals to the registration from the outside $(\gamma=2.667$ patients $/ \mathrm{min})$.

Then, the patient arrival process is proved whether to be the Poisson distribution by $\chi^{2}$ test. $H_{0}$ is: patient arrival process from the outside is the Poisson distribution, the distribution function is $F(x)=F_{0}(x)$, where $F_{0}(x)$ is the function for the Poisson distribution. Sample $x_{1}, x_{2}, \ldots, x_{n}$ extracted from the population $X$ divide $x$ axis into $k$ non-overlapping small intervals $\left(-\infty, t_{1}\right],\left(t_{1}, t_{2}\right], \ldots,\left(t_{k-1},+\infty\right)$, and the sample count is $f_{i}$ in $i_{t h}$ interval $\left(t_{i-1}, t_{i}\right]$. The critical value is $\chi_{0}^{2}=\sum_{i=1}^{k} \frac{\left(f_{i}-n p_{i}\right)^{2}}{n p_{i}}$, where $p_{i}=F_{0}\left(t_{i}\right)-F_{0}\left(t_{i-1}\right) \square$ Here, $f_{i}$ represents the observed count; $p_{i}$ represents the expected frequency; $n p_{i}$ represents the expected count. Given a significant level $\alpha$ and degree of freedom $k-s-1, \chi_{\alpha}^{2}(k-s-1)$ can be known from $\chi^{2}$ distribution table, here $s$ is the number of $x$ in $F_{0}(x)$. If $\chi_{0}^{2}<\chi_{\alpha}^{2}(k-s-1), H_{0}$ is accepted at the $5 \%$ significance level [6].

Considered patients actual arriving situation at the outpatient, 420 samples are divided into 9 intervals. $\chi_{0}^{2}=11.567$ is obtained by calculating the expected frequency and count (see Table 3). According to the significant level $\alpha=0.05$ and the degrees of freedom $7, \chi_{0.05}^{2}(7)=14.067$ is known from $\chi^{2}$ table, larger than $\chi_{0}^{2}$, so $H_{0}$ is accepted. Patients arrival process from the outside follows $\gamma=2.667$ patients $/ \mathrm{min}$ Poisson distribution.

The service time of each node (registration, consulting room, cashier, laboratory and pharmacy) for patients is randomly observed and recorded to calculate average service rate of each node. The service time of each node is separately certified whether to be the negative exponential distribution by $\chi^{2}$ test. For example, collect 103 samples of 
service time from the registration, and get the average service rate $\mu_{1}=1.397$ persons per minute, then process Exponential distribution of goodness of fit test. $H_{0}$ is assumed that the service process on the registration follows the Exponential distribution, Divide 103 samples into 5 intervals, and get the critical value $\chi_{0}^{2}=4.303$ (see Table 4). It is known that $\chi_{0.05}^{2}(3)=7.815$ at the $5 \%$ significant level and the degree of freedom 3 , larger than $\chi_{0}^{2}$. Therefore, $H_{0}$ is not rejected. The service time at the registration is Exponential distribution. Similarly, the service time on other nodes is testified to be Exponential distribution in the same way. The test results prove the service process of each node all follow the Exponential distribution (see Appendix). The service parameter of each node is summarized in Table 6.

Table 4. Service time on the registration of $\chi 2$ test statistics.

\begin{tabular}{|c|c|c|c|c|c|c|}
\hline $\mathbf{i}$ & $\begin{array}{c}\mathbf{x} \\
\text { (seconds) }\end{array}$ & $\mathbf{f}_{\mathbf{i}}$ & $\mathbf{p}_{\mathbf{i}}$ & $\mathbf{n p}_{\mathbf{i}}$ & $\mathbf{f}_{\mathbf{i}}-\mathbf{n} \mathbf{p}_{\mathbf{i}}$ & $\frac{\left(\boldsymbol{f}_{\boldsymbol{i}}-\boldsymbol{n} \boldsymbol{p}_{\boldsymbol{i}}\right)^{\mathbf{2}}}{\boldsymbol{n p}}$ \\
\hline 1 & $0-30$ & 46 & 0.503 & 51.765 & -5.765 & 0.642 \\
\hline 2 & $30-60$ & 31 & 0.250 & 25.749 & 5.251 & 1.071 \\
\hline 3 & $60-90$ & 16 & 0.124 & 12.808 & 3.192 & 0.795 \\
\hline 4 & $90-120$ & 7 & 0.062 & 6.371 & 0.629 & 0.062 \\
\hline 5 & 120 above & 3 & 0.061 & 6.306 & -3.306 & 1.733 \\
\hline & Total & 103 & 1.000 & 103.000 & 0.000 & 4.303 \\
\hline
\end{tabular}

Note: $i$ is each interval; $x$ is the arrival number per second.

\subsection{Calculate Arrival Rate of Each Node}

According to the information system of this hospital, patient proportion of every type in the population (i.e. the patient percentage of each route) is collected from previous data statistics. The external arrival rate of each route $\left(\gamma_{m}\right)$ is obtained based on the patient proportion as shown in Table 5.

Table 5. Average patient types.

\begin{tabular}{|c|c|c|}
\hline $\mathbf{m}$ & \% of Population & $\boldsymbol{\gamma}_{\mathbf{m}}$ \\
\hline Type I & $34.38 \%$ & 0.917 \\
\hline Type II & $8.75 \%$ & 0.233 \\
\hline Type III & $48.75 \%$ & 1.300 \\
\hline Type IV & $8.13 \%$ & 0.217 \\
\hline Total & 1 & 2.667 \\
\hline
\end{tabular}

The arrival rate of each node $\left(\lambda_{i}\right)$ is calculated by the equation set in Table 1 . The input parameters of each node including arrival rate, service rate and server number are concluded in Table $\mathbf{6}$.

\subsection{Establish Service Quality}

To establish service quality of current outpatient performance, the operation indicators of the queuing system are calculated on basis of the formulas in Table $\mathbf{2}$ by Matlab software. Matlab is an interactive software system for numerical calculation with a powerful data analysis such as dynamic system model, simulation and statistical analysis. This paper writes the corresponding program to the parameter in a standard M/M/c model by Matlab Software and makes statistical analysis with primary data collected in the field to validate the effectiveness of the model. The calculated results are listed in Table 7.

Table 6. Input parameters of each node.

\begin{tabular}{|c|c|c|c|}
\hline $\mathbf{i}$ & $\boldsymbol{\lambda}_{\mathbf{i}}$ & $\boldsymbol{\mu}_{\mathbf{i}}$ & $\mathbf{c}_{\mathbf{i}}$ \\
\hline Registration(1) & 2.667 & 1.397 & 3 \\
\hline Consulting Room (2) & 4.183 & 0.115 & 46 \\
\hline Cashier(3) & 3.733 & 1.044 & 5 \\
\hline Laboratory(4) & 1.517 & 0.167 & 10 \\
\hline Pharmacy(5) & 2.217 & 0.695 & 4 \\
\hline
\end{tabular}

Table 7 illustrates that the service system is not quite smooth from the view of the whole outpatient, and the service strength of each node is dramatically high. There is no free time for the staff on consulting room and laboratory node in term of the idle probability. The working pressure and strength of staff are both extraordinary high. The total patient 
number in queue and system separately reaches 11.378 and 65.449 persons per minute; and the total waiting time of one patient in queue and system reaches 6.287 and 24.076 minutes respectively.

From the view of one single node, the service condition on some nodes is reasonable, while the service status on other nodes is very terrible, such as consulting room, laboratory and pharmacy. In comparison to other nodes, Node 4 (laboratory) with ten servers at post produces inferior performance with the highest server utilization $(\rho=0.910)$ and no idle time $\left(P_{0}=0.000\right)$ for the staff. For the patients, queue length $\left(L_{q}=7.015\right)$, waiting time in queue $\left(W_{q}=4.625\right)$ and waiting time in the system $\left(W_{s}=10.625\right)$ on Node 4 are all the highest among the nodes. Node 1 (registration) with 3 servers at post provides relatively optimal performance of the system with the lowest server utilization $(\rho=0.636)$ and the biggest idle probability of staff $\left(P_{0}=0.126\right)$. At the same time, patients number in the system $\left(L_{s}=2.614\right)$, waiting time in queue $\left(W_{q}=0.264\right)$ and waiting time in the system $\left(W_{s}=0.980\right)$ are all the lowest compared with other nodes.

Table 7. Performance indicators of each node.

\begin{tabular}{|c|c|c|c|c|c|c|}
\hline $\mathbf{i}$ & $\mathbf{\rho}_{\mathbf{i}}$ & $\mathbf{P}_{\mathbf{0 i}}$ & $\mathbf{L}_{\mathbf{q i}}$ & $\mathbf{L}_{\mathbf{s i}}$ & $\mathbf{W}_{\mathbf{q i}}$ & $\mathbf{W}_{\mathbf{s i}}$ \\
\hline Registration(1) & 0.636 & 0.126 & 0.705 & 2.614 & 0.264 & 0.980 \\
\hline Consulting Room (2) & 0.789 & 0.000 & 0.319 & 36.615 & 0.076 & 8.753 \\
\hline Cashier(3) & 0.715 & 0.024 & 1.010 & 4.587 & 0.271 & 1.229 \\
\hline Laboratory(4) & 0.910 & 0.000 & 7.015 & 16.115 & 4.625 & 10.625 \\
\hline Pharmacy(5) & 0.797 & 0.028 & 2.328 & 5.518 & 1.050 & 2.489 \\
\hline Total & & & 11.378 & 65.449 & 6.287 & 24.076 \\
\hline
\end{tabular}

The indicators shown in Table 7 reflect a inappropriate operation status. For the patients, they need to visit the nodes back and forth in the outpatient and repeat to queue, under the condition of many waiting patients and much waiting time. If the patient needs the examination, he will stay in the outpatient for a considerable long time. For the staff, they need to continue working with the higher strength and little idle time. Under this situation with irritable patients and exhausted staff, it can well be imagined how difficult the relationship is between doctors and patients and how dissatisfied patients and staff are in this severe environment.

\subsection{Modify Servers Number}

To make an appropriate service process in the outpatient, there are two common measures to promote the service efficiency and quality. One way is to increase the service rate of the staff. This approach, based on traditional industrial engineering theory, can analyze the movement of employees through the qualitative and quantitative analysis, eliminate unnecessary and unreasonable action, make the most rational action and action sequence, and achieve the operation standardization. This approach is out of the scope of this research. This paper mainly studies another method to improve performance metrics by increasing the number of server desks [24].

To get a proper server number, the performance indicators can be as a series of variable function. In the queue model $\mathrm{M} / \mathrm{M} / \mathrm{c}, L_{q}$ is a function of $c, \rho$ and $p_{0}$, while $p_{0}$ is also a function of, $\rho, \lambda$ and $\mu$. So the function is too complicated to use the derivation method to calculate the proper $c$. In order to understand the influence of server number change on the queuing system, Node 5 is taken as an example. Fig. (3) and Fig. (4) demonstrate the extremely marginal changes for Node 5 with respect to patient number and waiting time in queue and system. Two aspects with this method should be considered simultaneously: one is the effect on the patients; the other one is the influence on the hospital. It is necessary to consider whether to satisfy the patients and whether to make the hospital to accept the change [25]. An optimal system performance is important for sustainability.

As shown in Fig. (3) and Fig. (4), when the server number is increased from four to five on Node 5, the performance indicators are greatly improved to some extent. The patient number in queue $\left(L_{q}\right)$ drops from 5.518 with four doctors to 3.693 with five doctors, while the patient number in the system $\left(L_{s}\right)$ drops from 2.328 to 0.503 . Similarly, the time in the system $\left(W_{s}\right)$ and waiting time in queue $\left(W_{q}\right)$ decrease to 1.666 and 0.227 respectively. In addition, the server utilization ( $\rho) 0.797$ on Node 5 with four servers changes to 0.638 with five servers. The idle probability of staff is changed from 0.028 to 0.038 . This means that patients spend less time in the queue and the system utilization becomes better. However, after the number of service desk increases to six, the performance improvement is not obvious. In other words when the number of servers is below a certain threshold, an outpatient can develop the performance by increasing the server number, whereas when it is slightly above this threshold, the performance has no change despite of the server increase [26]. Therefore, increasing one server to reach five servers for Node 5 is the optimal solution in this condition. 
It is not only can improve the satisfaction of patients in the outpatient, but also can increase the service efficiency.

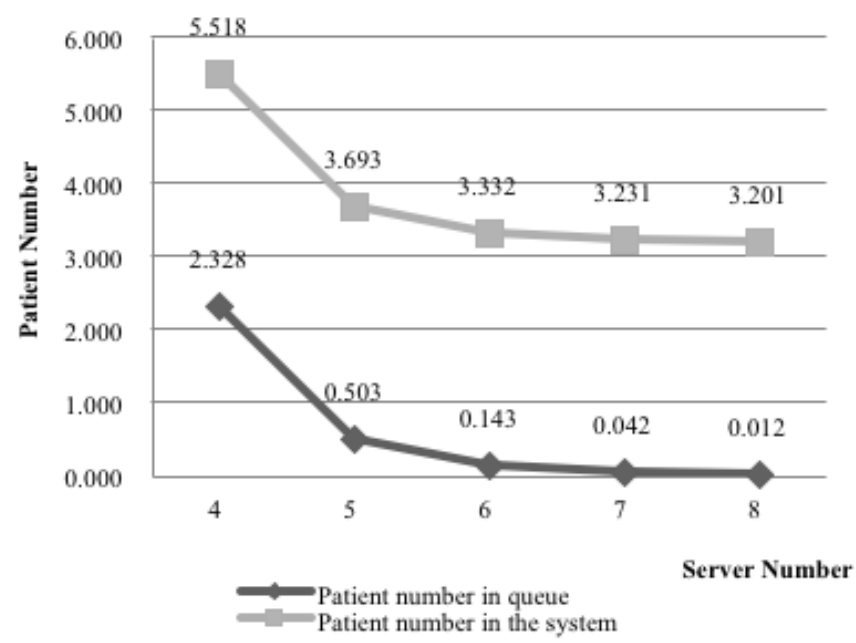

Fig. (3). Change of patient number in queue and in the system.

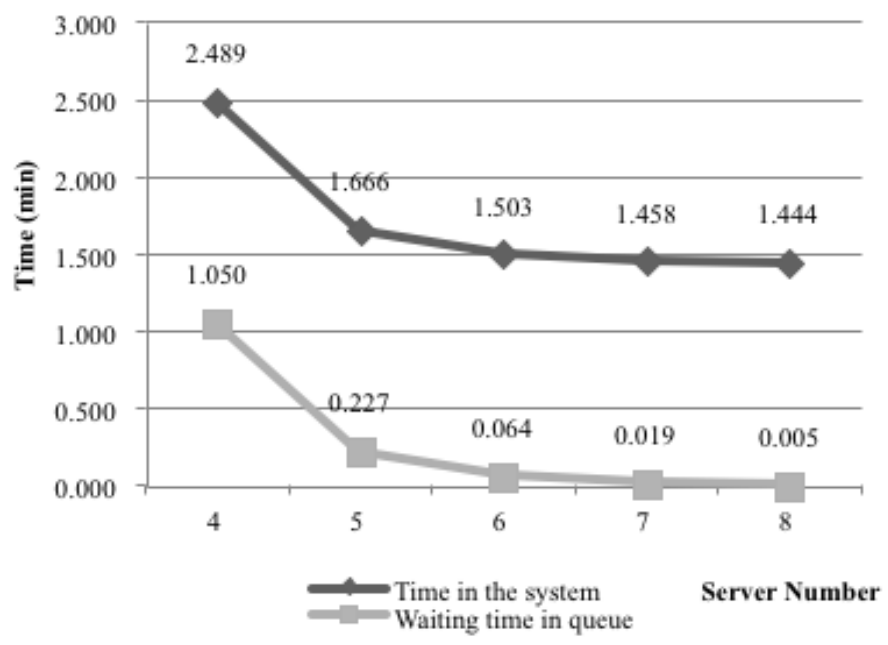

Fig. (4). Change of time in the system and waiting time in queue.

\section{CONCLUSION}

To sum up, this paper presents a new multi-type open queuing network model for outpatient process to guide the hospital manager decision corresponding to the actual demand in the outpatient. From a methodological perspective, this model is unique in taking the outpatient service process as a whole queuing network and considering the different patient types with different service orders among the service nodes. From a practical perspective, this quantitative model optimizes the allocation of server resources not only to increase patient satisfaction but also to improve the service efficiency on the occasion of limited resource and excessive demand. The adoption of queuing methodology can be important innovation in hospital management.

The limitation of this study is not considering the significantly difference in consulting room of different departments. For example, the patient number in the surgical department is usually larger than other departments in a general hospital. Although the servers on the nodes like registration or casher are almost the same, it is not rational to take various departments as several same servers. It will be considered that the various departments are taken as several single servers in parallel in the future research so that the analysis will be more accurate. The other limitation of this study is also not considering the differences in the average arrival rate of patients in different time ranges. The future work should consider regulating the service resource with real-time control so as to adapt to the patient demand change in practice. 


\section{APPENDIX}

The collected primary service time and $\chi^{2}$ test statistics for other service nodes except for the registration are listed from Table A.1-A.4.

Table A.1. Service time on the consulting room of $\chi^{2}$ test statistics.

\begin{tabular}{|c|c|c|c|c|c|c|}
\hline $\mathbf{i}$ & $\begin{array}{c}\mathbf{x} \\
\text { (minutes) }\end{array}$ & $\mathbf{f}_{\mathrm{i}}$ & $\mathbf{p}_{\mathrm{i}}$ & $\mathbf{n p} \mathbf{p}_{\mathbf{i}}$ & $\left(\mathbf{f}_{\mathrm{i}}-n p_{\mathrm{i}}\right)$ & $\frac{\left(f_{i}-n p_{i}\right)^{2}}{n p_{i}}$ \\
\hline 1 & $0-5$ & 39 & 0.438 & 44.677 & -5.677 & 0.721 \\
\hline 2 & $5-10$ & 25 & 0.246 & 25.108 & -0.108 & 0.000 \\
\hline 3 & $10-15$ & 20 & 0.138 & 14.110 & 5.890 & 2.458 \\
\hline 4 & $15-20$ & 11 & 0.078 & 7.930 & 3.070 & 1.189 \\
\hline 5 & 20 above & 7 & 0.100 & 10.175 & -3.175 & 0.990 \\
\hline & Total & 102 & 1.000 & 102.000 & 0.000 & 5.359 \\
\hline
\end{tabular}

Note: $i$ is each interval; $x$ is the arrival number per minute.

Table A.2. Service time on the cashier of $\chi^{2}$ test statistics.

\begin{tabular}{|c|c|c|c|c|c|c|}
\hline $\mathbf{i}$ & $\begin{array}{c}\mathbf{x} \\
(\text { seconds) }\end{array}$ & $\mathbf{f}_{\mathbf{i}}$ & $\mathbf{p}_{\mathbf{i}}$ & $\mathbf{n p}_{\mathbf{i}}$ & ${\left(\mathbf{f}_{\mathbf{i}}-\mathbf{n p}_{\mathbf{i}}\right)}$ & $\frac{\left(\boldsymbol{f}_{\boldsymbol{i}}-\boldsymbol{n} \boldsymbol{p}_{\boldsymbol{i}}\right)^{\mathbf{2}}}{\boldsymbol{n} \boldsymbol{p}_{\boldsymbol{i}}}$ \\
\hline 1 & $0-40$ & 43 & 0.501 & 47.634 & -4.634 & 0.451 \\
\hline 2 & $40-80$ & 28 & 0.250 & 23.750 & 4.250 & 0.761 \\
\hline 3 & $80-120$ & 14 & 0.125 & 11.841 & 2.159 & 0.393 \\
\hline 4 & $120-160$ & 7 & 0.062 & 5.904 & 1.096 & 0.203 \\
\hline 5 & 160 above & 3 & 0.062 & 5.871 & -2.871 & 1.404 \\
\hline & Total & 95 & 1.000 & 95.000 & 0.000 & 3.212 \\
\hline
\end{tabular}

Note: $i$ is each interval; $x$ is the arrival number per second.

Table A.3. Service time on the laboratory of $\chi^{2}$ test statistics.

\begin{tabular}{|c|c|c|c|c|c|c|}
\hline $\mathbf{i}$ & $\begin{array}{c}\mathbf{x} \\
\text { (minutes) }\end{array}$ & $\mathbf{f}_{\mathrm{i}}$ & $\mathbf{p}_{\mathrm{i}}$ & $\mathbf{n p}_{\mathbf{i}}$ & $\left(\mathbf{f}_{\mathrm{i}}-\mathbf{n} \mathbf{p}_{\mathrm{i}}\right)$ & $\frac{\left(f_{i}-n p_{i}\right)^{2}}{n p_{i}}$ \\
\hline 1 & $0-5$ & 61 & 0.565 & 62.194 & -1.194 & 0.023 \\
\hline 2 & $5-10$ & 30 & 0.246 & 23.344 & 6.656 & 1.898 \\
\hline 3 & $10-15$ & 12 & 0.107 & 10.145 & 1.855 & 0.339 \\
\hline 4 & $15-20$ & 5 & 0.046 & 4.409 & 0.591 & 0.079 \\
\hline 5 & 20 above & 2 & 0.036 & 3.389 & -1.389 & 0.569 \\
\hline & Total & 110 & 1.000 & 103.481 & 6.519 & 2.909 \\
\hline
\end{tabular}

Note: $i$ is each interval; $x$ is the arrival number per minute.

Table A.4. Service time on the pharmacy of $\chi^{2}$ test statistics.

\begin{tabular}{|c|c|c|c|c|c|c|}
\hline i & $\begin{array}{c}\mathbf{x} \\
\text { (minutes) }\end{array}$ & $\mathbf{f}_{\mathrm{i}}$ & $\mathbf{p}_{\mathrm{i}}$ & $n p_{i}$ & $\left(\mathbf{f}_{\mathrm{i}}-\mathbf{n} \mathbf{p}_{\mathrm{i}}\right)$ & $\frac{\left(f_{i}-n p_{i}\right)^{2}}{n p_{i}}$ \\
\hline 1 & $0-1$ & 42 & 0.501 & 49.092 & -7.092 & 1.025 \\
\hline 2 & $1-2$ & 33 & 0.250 & 24.500 & 8.500 & 2.949 \\
\hline 3 & $2-3$ & 14 & 0.125 & 12.227 & 1.773 & 0.257 \\
\hline 4 & $3-4$ & 5 & 0.062 & 6.102 & -1.102 & 0.199 \\
\hline 5 & 4 above & 4 & 0.062 & 6.079 & -2.079 & 0.711 \\
\hline & Total & 98 & 1.000 & 98.000 & 0.000 & 5.141 \\
\hline
\end{tabular}

Note: $i$ is each interval; $x$ is the arrival number per minute.

\section{CONFLICT OF INTEREST}

The authors confirm that this article content has no conflict of interest. 


\section{ACKNOWLEDGEMENTS}

This work was funded by Innovation Method of Ministry of Science and Technology of China (2012IM040500 and 2013IM030100) and the Doctoral Program of China's Higher Education (20120032110035).

\section{REFERENCES}

[1] J. Yang, and H. Yu, "Outpatient service flow optimization and effectiveness analysis", Chin. Hospital, vol. 13, no. 9, pp. 66-68, 2009. Available from: http://en.cnki.com.cn/Article_en/CJFDTOTAL-ZGYU200909028.htm

[2] X. Zhu, R. Miao, and Z. Jiang, Research and Implementation of Outpatient Appointment and Queuing Management System in Hospital Based on Lean Thinking. 2008. Available from: http://en.cnki.com.cn/Article_en/CJFDTotal-GYGC200806026.htm

[3] J. Xu, Y. Meng, Z. Shi, and Y. Jia, "Study and application status quo of process management in hospitals both at home and in abroad", Clin. Nurs. Res., vol. 24, no. 8B, pp. 2071-2073, 2010. Available from: http://en.cnki.com.cn/Article_en/CJFDTOTAL-SXHZ201023001.htm

[4] J. Cheng, Y. Zhang, L. Zhang, and S. Lu, "Analysis on the professional stress of doctors and nurses and the doctor-patient relationship", China Practical Medicine, vol. 5, no. 35, pp. 240-242, 2010. Available from: http://en.cnki.com.cn/Article_en/ CJFDTOTALZSSA201035206.htm

[5] C. Ma, and Y. Hou, "Factor and strategy for harmonious doctor-patient relationship", Science Time, no. 1, p. $281,2015$.

[6] "An application of discrete-event simulation to an outpatient healthcare clinic with batch arrivals", In: Proceedings of the 2011 Winter Simulation Conference. NY: Curran Associates, Inc., 2012.Phoenix, USA [http://dx.doi.org/10.1109/WSC.2011.6147839]

[7] P.T. Vanberkel, R.J. Boucherie, E.W. Hans, and J.L. Hurink, "Optimizing the strategic patient mix combining queueing theory and dynamic programming", Comput. Oper. Res., vol. 43, pp. 271-279, 2014. [http://dx.doi.org/10.1016/j.cor.2013.09.020]

[8] D. Kozlowski, and D. Worthington, "Use of queue modelling in the analysis of elective patient treatment governed by a maximum waiting time policy", Eur. J. Oper. Res., vol. 244, pp. 331-338, 2015.

[http://dx.doi.org/10.1016/j.ejor.2015.01.024]

[9] Y. Wu, and G. Du, Foundations of Management Science, Tianjin University Press: Tianjin, China, 2009. Available from: http://www.abebooks.com/Management-Science-Foundation-Case-Study-English/4629565226/bd

[10] C. Lakshmi, and A.I. Sivakumar, "Application of queueing theory in health care: A literature review", Oper. Res. Health Care, no. 2, pp. 25-39, 2013. [http://dx.doi.org/10.1016/j.orhc.2013.03.002]

[11] K.M. Mital, "Queuing analysis for outpatient and inpatient services: a case study", Manage. Decis., vol. 48, no. 3, pp. 419-439, 2010. [http://dx.doi.org/10.1108/00251741011037783]

[12] R. Mehandiratta, "Applications of queuing theory in health care", Int. J. Comput. Bus. Res., vol. 2, no. 2, 2011. Available from: http://www.researchmanuscripts.com/PapersVol2N2/IJCBRVOL2N2P9.pdf

[13] S. Fomundam, and J.W. Herrmann, "A survey of queuing theory applications in healthcare", Mech. Eng., no. 24, pp. 1-22, 2007. Available from: http://drum.lib.umd.edu/bitstream/handle/1903/7222/tr_2007-24.pdf?sequence=1"

[14] J.K. Cochran, and K.T. Roche, "A multi-class queuing network analysis methodology for improving hospital emergency department performance", Comput. Oper. Res., no. 36, pp. 1497-1512, 2009. [http://dx.doi.org/10.1016/j.cor.2008.02.004]

[15] N. Yankovic, and L.V. Green, "Identifying good nursing levels: A queuing approach", Oper. Res., vol. 59, no. 4, pp. 942-955, 2011. [http://dx.doi.org/10.1287/opre.1110.0943]

[16] H. Mi, "Analysis on queuing network of hospital outpatient service", J. Northwest Normal University, vol. 34, no. 2, pp. 25-31, 1998.

[17] P. Feng, Harbin Medical University Based on Queuing Theory Research on Optimization of Infection in a Hospital Outpatient Setting, 2012. Available from: http://globethesis.com/?t=2254330425965920

[18] W. Chang, Y. Peng, and S. Dong, "Queuing theory application to measure the efficiency of registration and cashier", Chin J. Hospital Admin, vol. 21, no. 12, pp. 810-814, 2005.

[19] W. Zhou, "Application Research of Queuing Theory Model in Medical Service System", Dissertation, HuaZhong University of Science and Technology, China, 2011.

[20] Q. Wang, "The Study on Optimizing Outpatient Service Process Based on Queuing Theory and Business Process Reengineering Theory", Dissertation, HuaZhong University of Science and Technology, China, 2008. Available from: http://www.dissertationtopic.net/doc/1556526

[21] W.L. Winston, Operations Research: Application and Algorithms. Tsinghua University Press: Beijing, China, 2006. Available from: https://mariellalimn.files.wordpress.com/2015/08/winston-operationsresearch.pdf

[22] N. Tian, X. Xu, and Z. Ma, Discrete-time Queuing Theory. Science Press: Beijing, China, 2008. [http://dx.doi.org/10.1287/opre.6.1.96]

[23] Q. Hu, Stochastic Operations Research. Tsinghua University Press: Beijing, China, 2012. 
[24] H. Takagi, "From computer science to service science: Queues with human customers and servers", Comput. Netw., vol. 66, pp. 102-111, 2014 [http://dx.doi.org/10.1016/j.comnet.2014.03.014]

[25] J. Goddard, and M. Tavakoli, "Efficiency and welfare implications of managed public sector hospital waiting lists", Eur. J. Oper. Res., vol. 184, pp. 778-792, 2008. [http://dx.doi.org/10.1016/j.ejor.2006.12.003]

[26] P. Gonzalez, and C. Herrero, "Optimal sharing of surgical costs in the presence of queues", Math. Methods Oper. Res., vol. 59, pp. 435-446, 2004

[http://dx.doi.org/10.1007/s001860400350]

(C) Mingzhu and Ershi; Licensee Bentham Open.

This is an open access article licensed under the terms of the Creative Commons Attribution-Non-Commercial 4.0 International Public License (CC BY-NC 4.0) (https://creativecommons.org/licenses/by-nc/4.0/legalcode), which permits unrestricted, non-commercial use, distribution and reproduction in any medium, provided the work is properly cited. 